\title{
On Approximation of Multivalued Solution for Hamilton-Jacobi equation
}

\author{
Ekaterina A. Kolpakova* \\ * Krasovskii Institute of Mathematics and Mechanics UrB RAS,Ural \\ Federal University, Yekaterinburg, Russia (e-mail: eakolpakova@ \\ gmail.com).
}

\begin{abstract}
The paper deals with the Cauchy problem for Hamilton-Jacobi equation with discontinuous w.r.t. state variable Hamiltonian. In this case we use the notion of M-solution proposed by Subbotin. We consider the sequence of auxiliary Cauchy problems for HamiltonJacobi equations with Lipschitz continuous w.r.t. phase variable Hamiltonians. We show that the sequence of distances between of graphs of solutions for auxiliary Cauchy problems and graph of M-solution tends to zero in metrics $L^{1}$.
\end{abstract}

(C) 2018, IFAC (International Federation of Automatic Control) Hosting by Elsevier Ltd. All rights reserved.

Keywords: Hamilton-Jacobi equation, multivalued solution, minimax/viscosity solution, convergence in $L^{1}$, viability set.

\section{INTRODUCTION}

The paper is concerned with the multivalued solutions for Hamilton-Jacobi equations and Hamiltonian is discontinuous w.r.t. phase variable function. Nowadays the theory of discontinuous Hamilton-Jacobi equations attracts a great attention (H.Ishii (1985), G.Barles and B.Perthame (1987)). First the theory of the Hamilton-Jacobi equations were studied for the smooth Hamiltonians (Crandall et al. (1992), Subbotin (1993)). Even in this case the solution of the Hamilton - Jacobi equation can be nonsmooth and only continuous function. When the Hamiltonian is only continuous function it is shown the solution should be considered in the class of multivalued mappings (Lakhtin and Subbotin (1998a)).

There are several approaches to definition of generalized solution for the Hamilton-Jacobi equations with the discontinuous Hamiltonian. Ishii (H.Ishii (1985)) introduced the viscosity approach. Subbotin (Lakhtin and Subbotin (1998a)) proposed the concept of M-solutions (multivalued solutions). This notion involves the viability property w.r.t. a differential inclusion. Once more approach uses the link with generalized solution of corresponding quasilinear equation (M.Coclite and N.Risebro (2007)). There are several existence and uniqueness theorems concerning the mentioned approaches.

Furthermore viscosity approach is equivalent the concept of M-solutions in the following sense: upper (low) envelope of the M-solution is the viscosity supersolution (subsolution) of the Hamilton-Jacobi equations (Lakhtin and Subbotin (1998b)). The Hamilton - Jacobi equations with the discontinuous Hamiltonians have application in optimal control problems, theory of hierarchical systems of the Hamilton-Jacobi equations (Kolpakova (2017)).

In the paper we examine the approximation of the solution of the Hamilton - Jacobi equations by the solutions of the smooth Hamilton - Jacobi equations. We construct the smooth Hamiltonian using the convolution. Note that the theory of smooth Hamilton - Jacobi equations is well-developed. Moreover one can approximate the solution of smooth Hamilton-Jacobi equations by parabolic equations or by the solution of the system of ordinary differential equations (Krasovskii and Kotelnikova (2010), Averboukh (2016)). This make it possible to use for the Hamiltonian - Jacobi equation with the discontinuous Hamiltonian the numerical methods developed in following works: Subbotin (1993), Kumkov et al. (2017),Falcone and Ferretti (1994), Quincampoix et al. (1999). We show the convergence to zero in space $L^{1}$ the Hausdorff distance between graphs of $\mathrm{M}$ - solution and continuous mini$\max /$ viscosity solutions. The general result is illustrated by the model example.

\section{STATEMENT}

In the paper we study the boundary Cauchy problem for the first order Hamilton-Jacobi equation

$$
\frac{\partial w(t, x)}{\partial t}+H(t, x, s)=0, \quad w(T, x)=\sigma(x)
$$

Here $t \in[0, T], x \in \mathrm{R}^{n}$.

We assume that

A1 Function $H$ is Lipschitz continuous w.r.t. $t, s$ and $H(t, \cdot, s) \in L^{1}\left(\mathrm{R}^{n}\right), H$ is Lipschitz continuous function w.r.t $x$ except the zero measure set of points of discontinuity.

A2 Function $|H(t, x, 0)| \leq \nu(1+|x|), \nu>0$ and

$$
\begin{aligned}
& \left|H\left(t, x, s_{1}\right)-H\left(t, x, s_{2}\right)\right| \leq \lambda(1+|x|)\left|s_{1}-s_{2}\right|, \\
& \forall(t, x) \in[0, T] \times \mathrm{R}^{n}, \lambda>0 .
\end{aligned}
$$

A3 Function $\sigma$ is continuously differentiable.

Let us introduce the Hamiltonian $H_{k}$ as convolution product:

$$
H_{k}(t, x, s)=\int_{\mathrm{R}^{n}} H(t, y, s) \eta_{k}\left(\frac{x-y}{\varepsilon_{k}}\right) d y=H * \eta_{k} .
$$


Here $\varepsilon_{k}>0, \lim _{k \rightarrow \infty} \varepsilon_{k}=0, \eta_{k}$ is mollifier. We assume that the family $\eta_{k}$ has the following properties

(1) $\eta_{k}(x)>0, \forall x \in \mathrm{R}^{n}$,

(2) $\int \eta_{k}(x) d x=1$,

(3) $\int_{|x|>\delta} \eta_{k}(x) d x \rightarrow 0$ as $k \rightarrow \infty, \forall \delta>0$.

Further we shall suppose that $\eta_{k} \in C_{0}^{\infty}$, the space of infinitely differentiable function with compact support. One can choose $\eta_{k}$ to be equal to

$$
\eta_{k}(x)=\left\{\begin{array}{r}
e^{-\frac{1}{1-\left|x / \varepsilon_{k}\right|^{2}}}, \text { if }|x|<\varepsilon_{k}, \\
0, \text { if }|x| \geq \varepsilon_{k} .
\end{array}\right.
$$

\subsection{Properties of Hamiltonian $H_{k}$}

Claim 1. Hamiltonian $H_{k}$ has properties

(1) The function $H_{k}$ is infinitely differentiable w.r.t. $x$.

(2) The function $H_{k}$ is sublinear w.r.t. $s$ and Lipschitz continuous w.r.t. $s$ with the Lipschitz constant $\lambda>0$.

(3) The function $H_{k}$ is Lipschitz continuous w.r.t. $x$ with the Lipschitz constant $\kappa>0$.

\section{Proof.}

1. Recall that if $H \in L^{1}\left(\mathrm{R}^{n}\right)$ and the approximate identity $\eta_{k} \in C_{0}^{\infty}$, then $H_{k}=H * \eta_{k}$ is infinitely differentiable w.r.t. $x$ (Resnick (1998)).

2. It is obvious that $H_{k}$ is Lipschitz continuous w.r.t. $s$. Let us calculate the Lipschitz constant of function $H_{k}$. Consider

$$
\begin{gathered}
\left|H_{k}\left(t, x, s_{1}\right)-H_{k}\left(t, x, s_{2}\right)\right| \leq \\
\int_{\mathrm{R}^{n}}\left|H\left(t, y, s_{1}\right)-H\left(t, y, s_{2}\right)\right| \eta_{k}\left(\frac{x-y}{\varepsilon_{k}}\right) d y \mid \leq \\
\lambda\left|s_{1}-s_{2}\right| \int_{\mathrm{R}^{n}}(1+|y|) \eta_{k}\left(\frac{x-y}{\varepsilon_{k}}\right) d y \leq \\
\lambda\left(1+|x|+\varepsilon_{k}\right)\left|s_{1}-s_{2}\right| \int_{\mathrm{R}^{n}} \eta_{k}\left(\frac{x-y}{\varepsilon_{k}}\right) d y \leq \\
\lambda\left(1+|x|+\varepsilon_{k}\right)\left|s_{1}-s_{2}\right| .
\end{gathered}
$$

The function $H_{k}$ is sublinear w.r.t. $x$. Indeed,

$$
\begin{gathered}
\left|H_{k}(t, x, 0)\right|=\left|\int_{\mathrm{R}^{n}} H(t, y, 0) \eta_{k}\left(\frac{x-y}{\varepsilon_{k}}\right) d y\right| \leq \\
\left|\int_{\mathrm{R}^{n}} \nu(1+|y|) \eta_{k}\left(\frac{x-y}{\varepsilon_{k}}\right) d y\right| \leq \\
\nu(1+|x|)\left|\int_{\mathrm{R}^{n}} \eta_{k}\left(\frac{x-y}{\varepsilon_{k}}\right) d y\right|=\nu(1+|x|) .
\end{gathered}
$$

3. Let us prove a Lipschitz continuity of $H_{k}$ w.r.t. $x$. Consider

$$
\begin{gathered}
\left|H_{k}\left(t, x_{1}, s\right)-H_{k}\left(t, x_{2}, s\right)\right|= \\
\left|\int_{\mathrm{R}^{n}} H(t, y, s) \eta_{k}\left(\frac{x_{1}-y}{\varepsilon_{k}}\right)-H(t, y, s) \eta_{k}\left(\frac{x_{2}-y}{\varepsilon_{k}}\right) d y\right| \leq \\
\left|\int_{\mathrm{R}^{n}} H(t, y, s) \dot{\eta}_{k}(\vartheta / \varepsilon) \frac{n}{\varepsilon_{k}}\right| x_{1}-x_{2}|d y| \leq K\left|x_{1}-x_{2}\right|,
\end{gathered}
$$

where $\kappa=\int_{C} H(t, y, s) \dot{\eta_{k}}(\vartheta / \varepsilon) \frac{n}{\varepsilon_{k}} d y, C$ is the support of $\dot{\eta}_{k} \in C_{0}^{\infty} ; H(t, y, s)$ is bounded under $y \in C$ and fixed $(t, s)$. Hence $H_{k}$ is Lipschitz continuous w.r.t. $x$.

\section{SOLUTION OF AUXILIARY PROBLEM}

Consider the Cauchy problem for the Hamilton-Jacobi equation with the Lipschitz continuous Hamiltonian

$$
\frac{\partial w_{k}(t, x)}{\partial t}+H_{k}\left(t, x, s_{k}\right)=0, \quad w_{k}(T, x)=\sigma(x) .
$$

Hamiltonian $H_{k}$ is defined by (2). Consider the sets.

$$
\begin{gathered}
E_{k}(t, x, s)=\left\{(f, g):|f| \leq \lambda\left(1+|x|+\varepsilon_{k}\right),\right. \\
\left.g=\langle f, s\rangle-H_{k}(t, x, s), \forall s \in \mathrm{R}^{n}\right\} \\
E(t, x, s)=\{(f, g):|f| \leq \lambda(1+|x|), \\
\left.\langle f, s\rangle-g \in\left[H_{*}(t, x, s), H^{*}(t, x, s)\right], \forall s \in \mathrm{R}^{n}\right\} .
\end{gathered}
$$

Here $H_{*}(t, x, s)=\liminf _{\xi \rightarrow x} H(t, \xi, s)$,

$$
H^{*}(t, x, s)=\limsup _{\xi \rightarrow x} H(t, \xi, s) .
$$

Remind the definition of a solution for problem (4) (Subbotin (1993)). A minimax/viscosity solution of problem (4) is a continuous function $w_{k}:[0, T] \times \mathrm{R}^{n} \rightarrow \mathrm{R}$ and gr $w_{k}$ is weakly viable set w.r.t. differential inclusion $(\dot{x}, \dot{z}) \in$ $E_{k}(t, x, s), E_{k}$ satisfies (5) and $w_{k}(T, x)=\sigma(x), \forall x \in \mathrm{R}^{n}$.

It follows from (Subbotin (1993)) that under assumptions A1-A3 there exists and unique the generalized solution of problem (4) in the class of continuous functions.

Remind the definition of a solution for problem (1) (Lakhtin and Subbotin (1998a)). The multivalued map $w:[0, T] \times \mathrm{R}^{n} \rightrightarrows \mathrm{R}$ is called an M-solution of problem (1), if gr $w$ is a maximal weakly viable set w.r.t.the differential inclusion $(\dot{x}, \dot{z}) \in E(t, x, s), E$ satisfies $(6)$ and $w(T, x)=\sigma(x), \forall x \in \mathrm{R}^{n}$.

From (Lakhtin and Subbotin (1998a))the M-solution of problem (1) exists and it is unique in the class of multivalued mappings.

We shall remind the following definition (Resnick (1998)). An upper limit of sequence of sets $\operatorname{Ls} w_{k}, k \rightarrow \infty$ is a set such that

$$
\mathrm{Ls} w_{k}=\left\{(t, x, z)=\lim _{i \rightarrow \infty}\left(t_{k_{i}}, x_{k_{i}}, z_{k_{i}}\right),\right.
$$

where $\left(t_{k_{i}}, x_{k_{i}}, z_{k_{i}}\right) \in$ gr $\left.w_{k_{i}}\right\}$.

Theorem 2. The M-solution of problem (1) satisfies the inclusion $\operatorname{Ls} w_{k} \subset$ gr $w, k \rightarrow \infty$, where $w_{k}$ is the minimax/viscosity solution of problem (4).

\section{Proof.}

Denote gr $w^{*}=\operatorname{Ls}$ gr $w_{k}$. At first we show that the set gr $w^{*}$ is not empty. Let $B \subset[0, T] \times \mathrm{R}^{n}$ be a compact and $(\tau, \xi) \in B$.

Consider $\zeta_{k}=w_{k}(\tau, \xi)$. By definition of the minimax solution gr $w_{k}$ is weakly invariant w.r.t. differential inclusion (5) for any $s \in \mathrm{R}^{n}$. Let $s=0$. From $\left(\tau, \xi, \zeta_{k}\right) \in$ gr $w_{k}$ there exists a solution $\left(x_{k}(\cdot), z_{k}(\cdot)\right)$ of differential inclusion (5) and $\left(T, x_{k}(T), z_{k}(T)\right) \in$ gr $w_{k}$. Thus $z_{k}(T)=\sigma\left(x_{k}(T)\right)$.

Remind that

$\dot{x}_{k} \leq \lambda\left(1+\left|x_{k}\right|+\varepsilon_{k}\right), \quad \dot{z}_{k}=H_{k}\left(t, x_{k}(t), 0\right) \leq \nu\left(1+\left|x_{k}(t)\right|\right)$. 
By Gronwall's lemma we have

$$
\begin{gathered}
\left|x_{k}(T)\right| \leq\left(1+\varepsilon_{k}\right)\left(e^{\lambda(T-\tau)}-1\right)+|\xi| e^{\lambda(T-\tau)} \leq \\
2 e^{\lambda(T-\tau)}+|\xi| e^{\lambda(T-\tau)},
\end{gathered}
$$

therefore

$$
\begin{gathered}
\left|w_{k}(\tau, \xi)\right|=\left|z_{k}(\tau)\right|=\left|\sigma\left(x_{k}(T)\right)+\int_{T}^{\tau} H_{k}\left(t, x_{k}(t), 0\right) d t\right| \leq \\
\left|\sigma\left(x_{k}(T)\right)\right|+\nu\left(1+\left|x_{k}(T)\right|\right)(T-\tau) \leq r .
\end{gathered}
$$

We get $\left|w_{k}(\tau, \xi)\right| \leq r$ for any $k$ and for any $(\tau, \xi) \in B$.

Now we prove that gr $w^{*}$ is weakly invariant w.r.t. differential inclusion (6). Let us choose $\left(t_{0}, x_{0}, z_{0}\right) \in$ gr $w^{*}$, $s \in \mathrm{R}^{n}$. By definition gr $w^{*}$ there exists a sequence $\left\{\left(\tau_{k}, \xi_{k}, \zeta_{k}\right)\right\} \in \operatorname{gr} w_{k}, \lim _{k \rightarrow \infty}\left(\tau_{k}, \xi_{k}, \zeta_{k}\right)=\left(t_{0}, x_{0}, z_{0}\right)$. The sets gr $w_{k}$ are weakly invariant w.r.t. differential inclusion (5), hence a solution of differential inclusion (5) exists and

$$
\left(x_{k}(\cdot), z_{k}(\cdot)\right):\left(t, x_{k}(t), z_{k}(t)\right) \in \text { gr } w_{k}, t \in\left[\tau_{k}, T\right] .
$$

The sequence $\left(x_{k}(\cdot), z_{k}(\cdot)\right)$ is uniformly bounded and equicontinuous. By Arzeli-Ascoli theorem we choose a convergent subsequence $\left(x_{k_{i}}(\cdot), z_{k_{i}}(\cdot)\right)$. Let us denote the limit

$$
\lim _{k \rightarrow \infty}\left(x_{k_{i}}(\cdot), z_{k_{i}}(\cdot)\right)=\left(x^{*}(\cdot), z^{*}(\cdot)\right) .
$$

The limit functions $\left(x^{*}(\cdot), z^{*}(\cdot)\right)$ are Lipschitz continuous. Therefore we can find such instant $\tau \in\left[t_{0}, T\right]$ that $\left(\dot{x}^{*}(\tau), \dot{z}^{*}(\tau)\right)$ exists. The sets $E_{k}, E$ are upper semicontinuous mappings, then for sufficient big number $K$ and small $\delta>0$ the following inclusion is valid

$$
E_{k}\left(t, x_{k}(t), s\right) \subseteq E\left(\tau, x^{*}(\tau), s\right)+B_{\alpha}, \quad \forall k \geq K
$$

$\forall t \in[\tau-\delta, \tau+\delta], \alpha>0$.

Let $\tau^{\prime} \in[\tau-\delta, \tau+\delta], \tau^{\prime} \neq \tau$. From the inclusion and convexity of $E\left(\tau, x^{*}(\tau), s\right)+B_{\alpha}$ we obtain

$$
\left(\frac{x_{k}\left(\tau^{\prime}\right)-x_{k}(\tau)}{\tau^{\prime}-\tau}, \frac{z_{k}\left(\tau^{\prime}\right)-z_{k}(\tau)}{\tau^{\prime}-\tau}\right) \in E\left(\tau, x^{*}(\tau), s\right)+B_{\alpha} .
$$

In the limit as $k \rightarrow \infty$ we get

$$
\left(\frac{x^{*}\left(\tau^{\prime}\right)-x^{*}(\tau)}{\tau^{\prime}-\tau}, \frac{z^{*}\left(\tau^{\prime}\right)-z^{*}(\tau)}{\tau^{\prime}-\tau}\right) \in E\left(\tau, x^{*}(\tau), s\right)+B_{\alpha} .
$$

$E\left(\tau, x^{*}(\tau), s\right)+B_{\alpha}$ is a closed set, consequently

$$
\left(\dot{x}^{*}(\tau), \dot{z}^{*}(\tau)\right) \in E\left(\tau, x^{*}(\tau), s\right)+B_{\alpha} .
$$

This inclusion is true for any $\alpha$, hence

$$
\left(\dot{x}^{*}(\tau), \dot{z}^{*}(\tau)\right) \in E\left(\tau, x^{*}(\tau), s\right) .
$$

This means that gr $w^{*}$ is weakly invariant w.r.t differential inclusion (6) and $w^{*}(T, x) \subset$ gr $\sigma(x), \forall x \in \mathrm{R}^{n}$. Thus we shew that gr $w^{*} \subset$ gr $w$.

\section{APPROXIMATION OF M-SOLUTION OF PROBLEM (4)}

Let us value the distance

$$
\begin{gathered}
\int_{[0, T] \times \mathrm{R}^{n}} \operatorname{dist}\left(w_{k}(t, x), w(t, x)\right) d x d t \leq \\
\int_{[0, T] \times \mathrm{R}^{n}} \operatorname{dist}\left(w_{k}(t, x), w^{*}(t, x)\right) d x d t .
\end{gathered}
$$

Here $\operatorname{dist}(\cdot, \cdot)$ denotes Hausdorff distance. Put $\tilde{w}$ is a measurable selector of the multivalued map $w^{*}$. Consider the expression

$$
\int_{[0, T] \times \mathrm{R}^{n}}\left|w_{k}(t, x)-\tilde{w}(t, x)\right| d x d t .
$$

We suppose that

$$
\lim _{k \rightarrow \infty} \int_{[0, T] \times \mathrm{R}^{n}}\left|w_{k}(t, x)-\tilde{w}(t, x)\right| d x d t=L>0 .
$$

Consider any compact set $C \subset[0, T] \times \mathrm{R}^{n}$. The quality is valid

$$
\lim _{k \rightarrow \infty} \int_{C}\left|w_{k}(t, x)-\tilde{w}(t, x)\right| d x d t=L>0 .
$$

Therefore

$$
\int_{C} \lim _{k \rightarrow \infty}\left|w_{k}(t, x)-\tilde{w}(t, x)\right|-L / \mu(C) d x d t=0 .
$$

Here $\mu(C)$ is the measure of the compact set $C$. By the definition $w(t, x)=\lim _{k \rightarrow \infty} w_{k}(t, x)$. Then

$$
\int_{C} \lim _{k \rightarrow \infty}\left|w_{k}(t, x)-\tilde{w}(t, x)\right|-L / \mu(C) d x d t<0,
$$

it is contradiction. Hence $L=0$.

\section{EXAMPLE}

We note that the mollifier can be nonsmooth function. Consider the example for this mollifier.

Let us consider the Cauchy problem with discontinuous Hamiltonian

$$
\frac{\partial w}{\partial t}+\frac{\partial w}{\partial x} \operatorname{sgn} x+\frac{1}{4}=0, \quad w(T, x)=x .
$$

Here $x \in \mathrm{R}, t \in\left[t_{0}, T\right]$. It is not difficult to check that the M-solution of this problem is the following multivalued map:

$$
w(t, x)= \begin{cases}-5 / 4(t-T)+x, & x>0 \\ {[3 / 4(t-T),-5 / 4(t-T)],} & x=0 \\ 3 / 4(t-T)+x, & x<0\end{cases}
$$

The right hand-side of the differential inclusion has the form

$E(t, x, s)=\{(f, g),|f| \leq 1, f s-g \in[-s+1 / 4, s+1 / 4]\}$.

Let us fix a point $\left(t_{0}, x_{0}, z_{0}\right)$ and $x_{0}>0$ then

$$
\dot{x}=1, \dot{z}=-1 / 4 \text {. }
$$

Integrating this system of the ordinary differential equations we get

$$
\begin{gathered}
x(t)=x_{0}+t-t_{0}, z(t)=z_{0}-1 / 4\left(t-t_{0}\right) . \\
\text { If } z_{0}=-5 / 4\left(t_{0}-T\right)+x_{0} \text { then } \\
z(t)=-t_{0}+5 / 4 T-1 / 4 t=-5 / 4(t-T)+x(t)-x_{0} .
\end{gathered}
$$

In the same way we consider another case. If $x_{0}<0$ then trajectories

$$
\dot{x}=-1, \dot{z}=-1 / 4
$$

are viable in graph $w$. Let $x_{0}=0, z_{0} \in\left[3 / 4\left(t_{0}-\right.\right.$ $\left.T),-5 / 4\left(t_{0}-T\right)\right]$. We choose

$$
\dot{x}=0, \dot{z}=0,
$$


then exists the instant $t_{1}$ such that for trajectories

$$
x(t) \equiv 0, z(t) \equiv z_{0}
$$

the following equality is valid

$$
z\left(t_{1}\right)=-5 / 4\left(t_{1}-T\right) \text { or } z\left(t_{1}\right)=3 / 4\left(t_{1}-T\right) .
$$

Further for $t \in\left[t_{1}, T\right]$ we go to the previous cases. These trajectories are viable in graph of the M-solution.

Let us construct the Hamiltonian $H_{k}$ :

$$
\begin{gathered}
H_{k}(t, x, s)=\frac{1}{d_{k}} \int_{B_{r_{k}}} H(t, x+y, s) d y= \\
s \frac{\left|x+r_{k}\right|-\left|x-r_{k}\right|}{2 r_{k}}+1 / 4 .
\end{gathered}
$$

Here $d_{k}$ denotes diameter of a ball with center at zero and radius $r_{k}$. In detailed the Hamiltonian $H_{k}$ has the form

$$
H_{k}(t, x, s)=\left\{\begin{array}{l}
s+1 / 4, \quad x \geq r_{k}, \\
\frac{x s}{r_{k}}+1 / 4,|x| \leq r_{k}, \\
-s+1 / 4, \quad x \leq-r_{k} .
\end{array}\right.
$$

The mollifier $\eta_{k}$ has the form

$$
\eta_{k}(x)=\left\{\begin{array}{l}
1, x \in B_{r_{k}}, \\
0, x \notin B_{r_{k}} .
\end{array}\right.
$$

The right hand-side of the differential inclusion in the definition of the minimax solution has the form

$$
E_{k}(t, x, s)=\left\{(f, g):|f| \leq 1, g=f s-H_{k}(t, x, s)\right\} .
$$

We consider the Cauchy problem with the Lipschitz continuous Hamiltonian

$$
\frac{\partial w_{k}}{\partial t}+H_{k}(t, x, s)=0, \quad w_{k}(T, x)=x .
$$

Let us design $a=e^{\frac{t-T}{r_{k}}}$. The minimax solution of this problem has the form

$$
w_{k}(t, x)= \begin{cases}-5 / 4(t-T)+x, & x \geq r_{k}, \\ -1 / 4 r_{k} \ln \frac{x}{r_{k}}-5 / 4(t-T) & \\ +5 / 4 r_{k} \ln \frac{x^{2}}{r_{k}}+r_{k}, & r_{k} a<x<r_{k}, \\ x e^{-\frac{t-T}{r_{k}}}-1 / 4(t-T), & |x| \leq r_{k} a, \\ -1 / 4 r_{k} \ln \frac{x}{-r_{k}}+3 / 4(t-T) & \\ -3 / 4 r_{k} \ln \frac{x^{-}}{-r_{k}}-r_{k}, & -r_{k}<x<-r_{k} a, \\ 3 / 4(t-T)+x, & x \leq-r_{k} .\end{cases}
$$

It is not difficult to check that the function $w_{k}$ is the minimax solution of problem (7).

Further we obtain the value

$$
\int_{\mathrm{R} \times[0, T]} \operatorname{dist}\left(w(t, x), w_{k}(t, k)\right) d x d t .
$$

It is obvious that in the domain $\{(t, x): t \in[0, T], x \in$ $\left.\left[r_{k}, \infty\right)\right\} w(t, x)=w_{k}(t, x)$.

In the domain $\left\{(t, x): t \in[0, T], x \in\left[r_{k} a, r_{k}\right]\right\}$ we get

$$
\begin{aligned}
& \int_{r_{k} a}^{r_{k}}-5 / 4(t-T)+x+1 / 4 r_{k} \ln \frac{x}{r_{k}}+5 / 4\left(t-r_{k} \ln \frac{x}{r_{k}}-T\right) \\
& \int_{r_{k} a}^{r_{k}}-r_{k} d x=-5 / 4(t-T) x+x^{2} / 2+1 / 4 r_{k}\left(x \ln x-x-x \ln r_{k}\right)
\end{aligned}
$$

$$
\begin{gathered}
-5 / 4(t-T) x+5 / 4 r_{k}\left(x \ln x-x-x \ln r_{k}\right)-\left.r_{k} x\right|_{r_{k} a} ^{r_{k}}= \\
-5 / 2(t-T) r_{k}-3 / 2 r_{k}^{2}+5 / 2 r_{k} a(t-T)+r_{k}^{2}\left(a^{2} / 2-a\right) \\
+3 / 2 r_{k}^{2} a\left(\frac{t-T}{r_{k}}-1\right) .
\end{gathered}
$$

In the domain $\left\{(t, x): t \in[0, T], x \in\left[-r_{k} a, r_{k} a\right]\right\}$ we get

$$
\begin{gathered}
\int_{0}^{r_{k} a}-5 / 4(t-T)+x-x a+1 / 4(t-T) d x= \\
-5 / 4(t-T) x+x^{2} / 2-x^{2} / 2 a+1 /\left.4(t-T) x\right|_{0} ^{r_{k} a}= \\
-5 / 4(t-T) a r_{k}+a^{2} r_{k}^{2} / 2(1-a)+1 / 4(t-T) a r_{k} .
\end{gathered}
$$

In the other domains the values

$$
\int_{\mathrm{R} \times[0, T]} \operatorname{dist}\left(w(t, x), w_{k}(t, k)\right) d x d t
$$

we obtain in the similar way. Hence we see the order of convergence

$$
\int_{\mathrm{R} \times[0, T]} \operatorname{dist}\left(w(t, x), w_{k}(t, k)\right) d x d t \approx C r_{k},
$$

where $C>0$.

\section{REFERENCES}

Averboukh, Y. (2016). Approximate solutions of continuous-time stochastic games. SIAM Journal on Control and Optimization, 54, 2629-2649.

Crandall, M.G., Ishii, H., and Lions, P.L. (1992). User's guide to viscosity solutions of second order partial differential equations. In Bulletin of the American Mathematical Society, volume 27, chapter 1, 1-67.

Falcone, M. and Ferretti, R. (1994). Discrete time highorder schemes for viscosity solutions of HamiltonJacobi-Bellman equations. Numerische Mathematik, 67, 315-344.

G.Barles and B.Perthame (1987). Discontinuous solutions of deterministic optimal stopping time problems. Mathematical Modelling and Numerical Analysis, 21, 557-579.

H.Ishii (1985). Hamilton - Jacobi equations with discontinuous hamiltonians on arbitrary open sets. Bulletin of the Faculty of Science and Engineering. Series I. Mathematics, 28, 33-77.

Kolpakova, E. (2017). A construction of nash equilibrium based on system of Hamilton-Jacobi equations of special type. Mat. Teor. Igr Pril, 9, 39-53.

Krasovskii, N. and Kotelnikova, A. (2010). An approachevasion differential game: Stochastic guide. Proceedings of the Steklov Institute of Mathematics, 269, 191-213.

Kumkov, S., LeMenec, S., and Patsko, V. (2017). Zero-sum pursuit-evasion differential games with many objects: Survey of publications. Dynamic Games and Applications, 7, 609-633.

Lakhtin, A.S. and Subbotin, A.I. (1998a). Multivalued solutions of first-order partial differential equations. Mat. Sb., 189, 33-58.

Lakhtin, A. and Subbotin, A. (1998b). Minimax and viscosity solutions of discontinuous first-order partial differential equations. Doklady Akademii Nauk, 359, 452.

M.Coclite and N.Risebro (2007). Viscosity solutions of hamilton-jacobi equations with discontinuous coefficients. J. Hyperbolic Differ. Equ, 4, 771-795. 
Quincampoix, M., Cardaliaguet, P., and Saint-Pierre, P. (1999). Numerical methods for differential games. In M. Bardi, T. Raghavan, and T. Parthasarathy (eds.), Stochastic and differential games: Theory and numerical methods. Annals of the international Society of Dynamic Games, 177-247. Birkhauser.

Resnick, S. (1998). A Probability Path. Birkhuser, Boston.

Subbotin, A. (1993). Generalized Solutions of FirstOrder PDEs: the Dynamical Optimization Perspective. Birkhauser, Boston-Berlin. 